\title{
The House Imagery Test: A New Measure of Mental Status
}

\author{
Yuan Yuan ${ }^{1}$, Xiaoxia Yu ${ }^{2}$, Hongchuan Zhang ${ }^{1, ~ * ~}$ \\ ${ }^{1}$ Department of Psychology, School of Sociology and Psychology, Central University of Finance and Economics, Beijing, China \\ ${ }^{2}$ Mental Health Education Center, Students' Affairs Department, North China Electric Power University, Beijing, China
}

Email address:

jackhczhang@gmail.com (Hongchuan Zhang)

${ }^{*}$ Corresponding author

\section{To cite this article:}

Yuan Yuan, Xiaoxia Yu, Hongchuan Zhang. The House Imagery Test: A New Measure of Mental Status. Psychology and Behavioral Sciences. Vol. 10, No. 1, 2021, pp. 49-55. doi: 10.11648/j.pbs.20211001.16

Received: January 21, 2021; Accepted: January 28, 2021; Published: February 2, 2021

\begin{abstract}
Imagery Communication Psychotherapy (ICP), a Chinese native psychotherapy, uses imagery as a medium to communicate in depth with clients. As the most essential and widely used imagery in ICP, house imagery had been found efficient to reveal global mental status. On this basis, the House Imagery Test (HIT), a new projective test, was developed. This article reports the development and validation of the HIT on a survey of 478 undergraduate students. Symptom Checklist 90 (SCL-90), Self-rating Depression Scale (SDS), Self-rating Anxiety Scale (SAS), Positive Affect and Negative Affect Scale (PANAS), and Trait Coping Style Questionnaire (TCSQ) were used as criterion in the current study. The results showed that the HIT had good test-retest reliability $(r=.69)$ and internal consistency $(r=.79)$. Exploratory factor analysis of the HIT exhibited a 4-factor structure, which was verified by further confirmatory factor analysis. The HIT also correlated significantly with SCL-90, SDS, SAS, PANAS and TCSQ. Overall, the HIT had acceptable reliability and validity. It was proved to be a novel and solid projective test for measurement of mental status. These findings provide a new tool to aid the clinic practice and suggest a new technical route for the development of projective tests.
\end{abstract}

Keywords: Projective Test, Imagery Communication Psychotherapy, Imagery, The House Imagery Test

\section{Introduction}

Psychological tests, bridging psychological theories and practice, are widely used in various sections of clinical practice including assessment, screening, diagnoses etc. These tests can be divided into three major types: self-report scale, projective scale and situational scale. It is of great value for psychologists and psychotherapists to choose an appropriate measurement for a certain goal.

Self-report scale is the mainstream psychometric instrument by providing standard procedures and convenience to score and interpret the results. Meanwhile, classical projective scales are also popular as deeply influenced by the climate of psychoanalysis [1]. Rorschach Inkblot Test (RIT), House-Tree-Person Test (HTP) and Thematic Apperception Test (TAT) were listed among the most popular instruments (rated among the top 10) used by clinic psychologists for several decades $[1,2]$.

The concept of projective method originated from psychoanalytic school. Frank, the first person who systematically introduced projective method, defined it as a technique to measure mental status based on one's interpretation on "vague or ambiguous unstructured stimuli" [3]. He clarified the procedure as following: a) presenting target stimuli (usually with ambiguous meanings) to the participants; b) asking the participants interpret or organize the stimuli; c) assessing the projected attitudes, desires, emotions and personalities based on their interpretation and organization. Such an approach was believed to accurately and thoroughly present the individual's inner world. Moreover, this kind of scales can effectively avoid some response tendencies like self-concealment and social desirability that are common flaws in traditional self-report scales.

However, projective scales have been heavily criticized as their reliability and validity usually fail to meet the psychometric standards. Taking Rorschach Inkblot Test (RIT), the most widely used projective scale in the world, as example, it called forth sharp criticism because of its strong subjectivity together with low reliability and validity. This situation did not change until the 1970s when Exner established the standardized 
norm and developed the Comprehensive System, leading RIT to a more objective and scientific position [4]. From then on, RIT has gained more popularity. Even though, projective scales like RIT are still criticized for other drawbacks including complicated scoring and interpretive systems, high cost of diagnosis and strict requirement for clinic experience.

It is of great importance to accurately assess the individual's mental status in psychological researches and clinical fields. Especially, the increasing amount and complication of psychopathological phenomena cast more challenges to mental measurements. Psychologists and psychotherapists are in need of scales that are convenient, low cost with satisfactory psychometric properties. Tong [5] pointed out that it might be an effective approach to innovate and develop projective scales.

The House Imagery Test (HIT) developed in the present study is a new projective test based on Jungian Psychology and Imagery Communication Psychotherapy [6]. It is heavily rooted in the clinical practice. ICP, invented by Chinese psychologist Jianjun Zhu in the 1990s, is a method of psychological counseling and psychotherapy with which the therapist and the client communicate with the symbolic of images at a subconscious level [7]. ICP was greatly influenced by Western psychology such as Psychoanalysis and Humanistic Psychology, and it also integrated Chinese Buddhism and Taoism. With imageries as the medium, the psychotherapist and client use a communication mode of "subconscious to subconscious" [7]. In other words, the psychotherapist communicates with the client by using the psychological symbolic meaning of the imagery, thus to help the client resolve negative emotion, eliminate complex, explore self deeply and integrate personality, so as to promote the individual's self-growth and development. The past twenty years have witnessed a tremendous growth in the use of ICP and its clinical efficacy. In recent years, some empirical studies have documented the efficacy of ICP on treating neurosis including phobia, depression and obsessive-compulsive disorder, and in interventions for particular groups such as drug abusers and prisoners [8-11].

House imagery is the most essential and widely used imagery in ICP. Using this technique, the therapist instructs the client into a totally relaxed situation and leads the client to see the imagined "house" (symbolic of one's mental world). In this way, the therapist would be able to assess the client's emotion styles, personalities and the present mental status based on the details of the house like color, structure, openness etc. Clinical practice has showed that the house imagery technique can illustrate a complete picture of the client's mental status and help to get a primary evaluation whether the client has any mental disorder.

However, given its effectiveness, this procedure of the house imagery communication is often not understood by novice. A possible solution to this limit is to develop a standardized projective test. Through this effort, we can offer a novel projective measurement supplementing the existing self-report scales and traditional projective tests. On the other hand, a standardized projective test can be easily turned into software, and can thus be used in large-scale surveys and cross-cultural studies.
In the current study, we attempted to develop an imagery projective test, the House Imagery Test (HIT), and to verify its reliability and validity. Based on the aforementioned literature and reasoning, we assumed that house imagery communication could be transformed into a standardized projective test, i.e., the House Imagery Test. Items of the HIT were all designed according to symbolic meanings of house imagery. It is reasonable to hypothesize that the HIT has satisfactory reliability and validity, and it could accurately assess the test-taker's global mental health.

\section{Method}

\subsection{Participants}

A total of 478 Chinese undergraduate students from 3 public elective courses in two semesters were recruited in the current study. 117 of them participated in the autumn semester ( 83 females, 34 males; $M_{\text {age }}=19.27$ years, $S D=$ 1.16), and 361 participated in the spring semester (245 females, 116 males; $M_{\text {age }}=18.70$ years, $S D=1.15$ ), with a 6-months gap. Data analysis showed no difference on gender or age between those two groups. 192 students from the spring semester were retested one month later (133 females, 59 males; $M_{\text {age }}=18.55$ years, $S D=1.10$ ). At each time point, the participants were group-tested in quiet classrooms and were told that they would complete an anonymous survey and get course credits for participation.

\subsection{Instruments}

\subsubsection{The House Imagery Test}

The HIT used in present study is composed of 12 items that are all core questions during the house imagery communication. These items, covering varieties of properties like texture, function, appearance, cleanliness, light, are all highly related to the test-taker's mental status according to clinical practice of ICP.

In order to meet the psychometric standards, these 12 items are all single-choice questions. In the pilot study, the original scale was presented to ICP experts to develop a scoring system. Options of each item were firstly ranked by mental health level according to the clinic experience. Then each option was given a certain score ranging from 1 to 5 (higher score indicating healthier mental state). The score of all the 12 items are aggregated to indicate the test-taker's global mental status.

\subsubsection{Criteria Scales}

The HIT was thought effective to assess global mental status, reflecting the individual's personality tone and especially the recent mood [6]. To this end, the following measurements were thus selected as criterion: Symptom Checklist 90 (SCL-90), Self-rating Depression Scale (SDS), Self-rating Anxiety Scale (SAS), Positive Affect and Negative Affect Scale (PANAS), and Trait Coping Style Questionnaire (TCSQ).

Symptom Checklist 90 (SCL-90). The present study used a Chinese version of SCL-90 [12]. The 90-item scale measures psychopathology using 10 primary symptom dimensions (somatization, compulsion, interpersonal sensitivity, depression, 
anxiety, hostility, phobia, paranoid, schizophrenia, and others). Participants responded on a 5-point scale ranging from 1 (not at all) to 5 (extremely), with higher scores indicating more serious psychopathology symptoms $(\alpha=.62 \sim .88)$.

Self-rating Depression Scale (SDS). The Chinese version of SDS, originated from Zung et al. [13], uses 20 questions to measure the individual's depression level [12]. Responses range from 1 (a little of the time) to 4 (most of the time), with higher scores indicating higher level of depression $(\alpha=.67)$.

Self-rating Anxiety Scale (SAS). SAS, developed by Zung [14], are widely used self-rated anxiety scales that consist of 20 questions, each with answers in a Likert-scale format rated from 1 (a little of the time) to 4 (most of the time). The Chinese version of SAS was used in this study [12]. Its internal consistency reliability was .69 in this study.

Positive Affect and Negative Affect Scale (PANAS). The Chinese version of PANAS [15], originated from Watson, Clark, and Tellegen [16], was used in the current study. This scale consists of 20 items, with 10 items for positive affect and 10 items for negative affect. Each item can be rated from 1 (not at all) to 5 (very much). The internal consistency reliability of positive affect and negative affect were .83 and .80 , respectively.

Trait Coping Style Questionnaire (TCSQ). The TCSQ was developed by Chinese scholars to reflect the individual's attitudes and behaviors in the face of adversity, and to present the trait-specific and health-related coping styles. This scale consists of 10 items related to positive coping styles and 10 for negative coping styles. Participants responded on a 5-point scale ranging from 1 (not at all) to 5 (extremely), with higher score on corresponding factor indicating more positive or negative coping styles $(\alpha=.59, .64)$.

\subsection{Procedure}

Before the test, participants were informed that their responses would be kept confidential and that they were free to withdraw from the study at any time. They were instructed to complete the HIT at first. In the HIT, instructions were given at the beginning as following:

Thank you for participating in our study! Please turn off your cell phone so as not to be disturbed. Now slow down your breathing, inhaling deeply and exhaling slowly. Do such a slow and deep breathing for two more times. With breaths slowing down, you will feel totally relaxed and very comfortable from top to toe.

Now imagine that you have moved from where you are now to a house. Maybe this house has ever appeared in the real world or in your imagination. Please answer the following questions about your house.

After filling out the HIT, participants moved on to complete criteria scales including SCL-90, SDS, SAS, PANAS, and TCSQ. Demographic data (e.g. gender, age) was also collected at the end of the test. It took about 40 minutes to finish the whole test.

\subsection{Data Analysis}

SPSS 20.0 and Mplus 7.0 were used for data analysis. The data collected in the fall semester was used for exploratory factor analysis and the data collected in the spring semester was used for confirmatory factor analysis.

\section{Results}

\subsection{Exploratory Factor Analysis}

The principal component analysis with varimax rotation was used for factor analysis. KMO and Bartlett's test of sphericity showed that exploratory factor analysis was appropriate for the data $\left(\mathrm{KMO}=.78, \chi^{2}=473.86, d f=66, p\right.$ $<.001)$. There were 4 factors with eigenvalues greater than 1 after an orthogonal rotation. The four-factor solution accounted for $67.05 \%$ of the variance. Factor contribution rates of each factor were all above $10 \%$, as shown in Table 1 . Factor loading of each item is above .50, as shown in Table 2.

Table 1. Eigenvalue and contribution rate of each factor.

\begin{tabular}{llll}
\hline Factor & Eigenvalue & Contribution rate (\%) & Cumulative contribution rate (\%) \\
\hline 1 & 2.94 & 24.47 & 24.47 \\
2 & 2.05 & 17.09 & 41.56 \\
3 & 1.61 & 13.40 & 54.96 \\
4 & 1.45 & 12.09 & 67.05 \\
\hline
\end{tabular}

Table 2. Obliquely rotated factor loadings of HIT items.

\begin{tabular}{|c|c|c|c|c|c|}
\hline No. & Item description & Factor 1 & Factor 2 & Factor 3 & Factor 4 \\
\hline 4 & existence of the window & .87 & & & \\
\hline 7 & how difficult to open the window & .84 & & & \\
\hline 5 & openness of the window & .81 & & & \\
\hline 6 & having dust on the window or not & .78 & & & \\
\hline 9 & cleanliness of the house & & .80 & & \\
\hline 3 & appearance condition of the house & & .73 & & \\
\hline 8 & light in the house & & .73 & & \\
\hline 2 & function of the house & & & .67 & \\
\hline 10 & regularity in the house & & & & .78 \\
\hline 12 & other objects in the house & & & & .59 \\
\hline 11 & type of the playing TV program & & & & .52 \\
\hline
\end{tabular}


According to imagery characteristics reflected on items of each factor, the 4 factors can be labeled as window, appearance, material, and internal articles:

Factor 1: window. This factor can reflect the individual's connection with the outside world, motivation to explore the inner world, and the willingness of self-expressing and acceptance. It has 4 items about the window of the house, including having windows or not, dust on the window(s) and openness of them.

Factor 2: appearance. This factor can reflect the person's susceptibility to mental disorders. There are 3 items about cleanliness, appearance and light of the house.

Factor 3: material. This factor can reflect the person's most essential characteristic. There are 2 items about texture and function of the house.

Factor 4: internal articles. This factor can reflect if there is any subconscious conflict and how serious it is. There are 3 items about regularity of the house, the playing TV program, and some unusual objects like skeleton and alien.

\subsection{Reliability}

The internal consistency of the whole scale was supported by Cronbach's $\alpha$ coefficient of .79. The internal consistency coefficient of each subscale was .74, .62, .46 and .51, respectively. Correlations between four factors were .24 to .37 , and there were moderate to high correlations between scores on each factor and the total scale $(r=.61 \sim .86)$. The consistency of all four factors was .59.

A sub-sample of 192 participants completed the measure a month after the first administration to assess test-retest reliability. This sub-sample was not significantly different from the larger sample on any demographic measure. The test-retest reliability coefficient of the total score was .69 ( $p$
$<.01)$. These results showed that the HIT has adequate internal consistency and cross-time stability.

\subsection{Validity}

\subsubsection{Construct Validity}

The four dimensions of the HIT were included in a structure model on Mplus 7.0 to establish the scale's construct validity. The 12-item first-order factor model resulted in a satisfactory model fit, $\chi^{2}=74.86, d f=41, \chi^{2} / d f=1.83, \mathrm{CFI}=.97$, TLI $=.95$, $\mathrm{SRMR}=.04$, $\mathrm{RMSEA}=.05$.

\subsubsection{Criterion-related Validity}

Correlations between HIT factors and criteria scales are presented in Table 3. HIT was negatively correlated with somatization, depression, hostility and schizophrenia of SCL-90 ( $\mathrm{r}$ values range from -.13 to -.20 , all $\mathrm{p}$ value $<.05$ ), SAS $(r=-.13, p<.05)$, and negative affect of PANAS $(r=-.21$, $p<.01)$. It also had a marginal negative correlation with SDS $(r=-.12, p<.07)$. As for dimensions of HIT, the factor Window was negatively correlated to somatization, hostility and schizophrenia of SCL-90, SAS, and negative affect of PANAS $(r=-.13 \sim-.24, p<.05)$. The factor appearance was negatively correlated to SDS, SAS, negative coping style, and most factors of SCL-90 $(r=-.12 \sim-.22, p<.05)$. It also had a marginal negative correlation with negative affect $(r=-.13, p$ $<.07$ ), and a marginal positive correlation with positive coping styles $(r=.13, p<.07)$. The factor material only had a negative correlation with negative affect of PANAS $(r=-.19, p<.01)$. Internal article of HIT had a significant negative correlation with somatization and schizophrenia $(r=-.12,-.12, p<.05)$, and a marginal negative correlation with hostility $(r=-.11, p$ $<.07)$ of SCL-90. Meanwhile, it was positively correlated to positive coping style $(r=.18, p<.01)$.

Table 3. Correlations between the HIT factors and criteria.

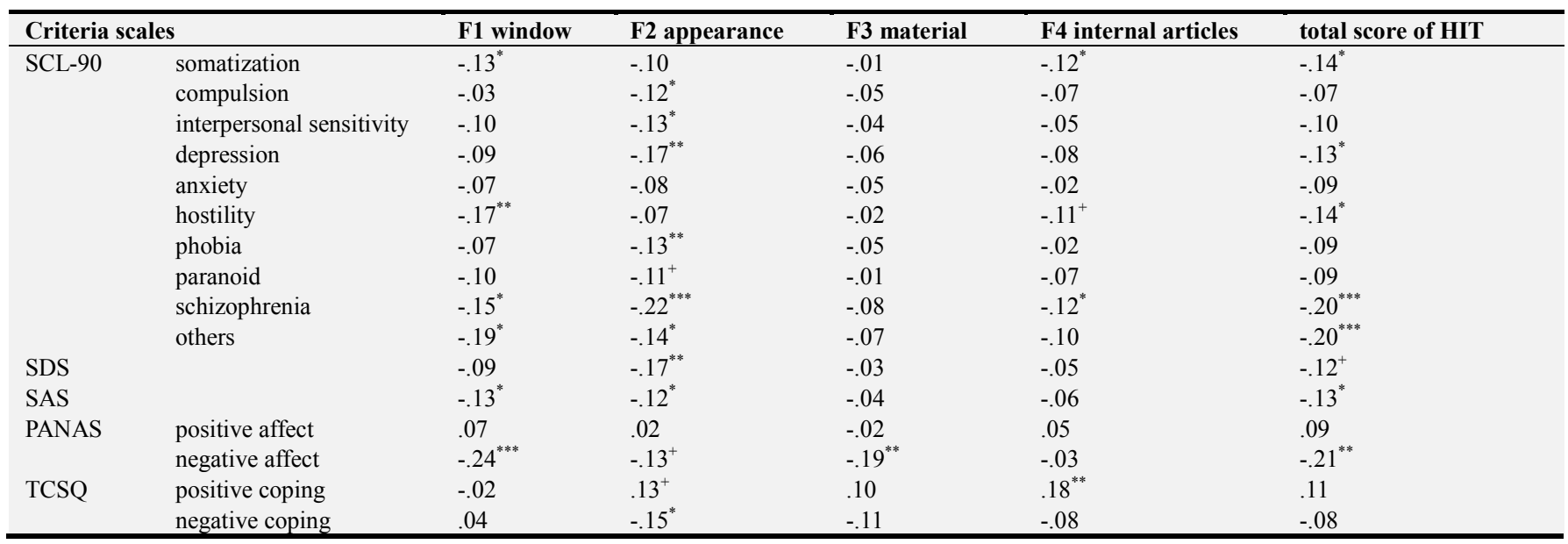

Note. ${ }^{*} p<.05,{ }^{* *} p<.01,{ }^{* * *} p<.01,{ }^{+} p<.07$.

\section{Discussion}

The present study transformed a typical clinic practice, the house imagery communication into a standardized projective test. This transformation is essential due to that in the clinic settings, the interpretation of the unique meaning of the house imagery was heavily influenced by the personal skill of the psychotherapist. In the present study, we developed the HIT as 
a new measure for global mental health evaluation. With its scoring system explicitly expressed based on the clinic meanings of the imagery features, psychologists of different backgrounds can use it for primary evaluation, without knowing the exact method to reach the meaning of a certain imagery feature.

\subsection{Reliability and Validity of the HIT}

The HIT consists of 12 items, with a 4-factor structure (window, appearance, material, and internal articles) resulted from exploratory factor analysis and further confirmed by confirmatory factor analysis. The structure of the test is consistent with the research expectations, and in line with the extensive clinical experience of house imagery technology in ICP. The internal consistency coefficient of HIT was .79, and the test-retest reliability was .69. There were low to moderate correlations between factors $(r=.24 \sim .37)$, and moderate to high correlations between each factor and total score of the test $(r=.61 \sim .86)$. These results showed that the HIT had adequate measurement reliability and validity.

Each factor of the HIT relates to a feature of house imagery and represents a certain side of mental status. Window of the HIT can reflect the individual's motivation to explore the inner world, and the willingness of self-expressing and acceptance. If there is no window, or windows are closed and it's difficult to open, or they are dusty, the test-taker may be more self-reclusive and have lower mental health level. The "appearance" dimension is closely related to the clinical evaluation for it can reflect the individual's current emotions and inner feelings. For example, the individual whose "house" is dilapidated or has little light inside is more likely to be inferior and depressed. The "material" dimension refers to texture and function of the house. It reflects the individual's relatively stable characteristic. The last dimension "internal articles", revealing the test-taker's subconscious status, is significant for clinic evaluation to identify the existence and extent of mental problems.

In accordance with the proposed meaning of the house imagery, the HIT correlated well with SCL-90, SDS, SAS, PANAS, and TCSQ as expected. It showed that the HIT can reflect one's global mental health status and indicate one's specific emotional tone and coping style. However, either the HIT or its dimension showed moderate to low correlations with criteria scales. A possible reason may be the discrepancy on measurements between projective test and self-rating test. The HIT evaluates mental health from the perspective of implicit psychological activity while these criteria scales take the perspective of differences on external behaviors. Shweder and D'Andrade [17] proposed a systematic distortion hypothesis, that self-rating scales measured the individual's behaviors based on his own memory. In that way, the subconscious content is missed. Meanwhile, criteria such as SCL-90, SDS, SAS are diagnosis scales. Some mild mental content reflected by the HIT may not be detected by these scales. In fact, the criteria validation has been found to be a common challenge in related studies [18]. After all, the correlation between the HIT and the criteria scales were significant and acceptable.

Generally speaking, the HIT is neither a single personality measure nor a simple mental health scale. It is, however, a measure for global mental status. It employs a dual-track approach to evaluate both the individual's stable characteristic related with his current mental status (the dimension "material") and the present mental health level (the dimension "window", "appearance", and "internal articles"). This kind of evaluation has been proved to be more instructive for further counseling and treatment.

\subsection{Innovation and Practical Significance of HIT}

The development of the HIT benefited from rich practical experience of Imagery Communication Psychotherapy. The HIT transformed that experience into a scientific and operational measure. It is different from self-rating scales due to its deep root in subconscious imagery interpretation, and is also more innovative than traditional projective tests due to its well-established psychometric indicators.

As Jain et al. [19] had noted, regarding sharp distinctions between projective and objective measures, the subject's task on commonly used personality tests injects considerable objectivity into many projective methods. The HIT uses the form of questionnaire to collect data, however, it is essentially different from self-reported tests. First of all, the HIT is a projective test that evaluates the individual's mental status by projecting it on house imagery. What's more, this test collects the symbolic meanings and implicit mental-related information of the imagery features but not external behaviors. Finally, the HIT employs a process evaluation pattern and can reflect consistent psychological characteristics.

As an innovative projective test, the HIT can accurately reflect individual's mental development status, mental health level and the deep reasons behind, from the perspective of the test-taker's imagery projected by subconscious activities, the symbolic of images and correlations between them. Compared with existing tests, it has some advantages as below.

First, it has a relatively clear structure and standardized testing procedure. It can be used without any special tools, complicated instructions, or even without testers. Thus, the influence of the tester's participation is greatly reduced. Second, the imagery is implicit and can arouse the interest of the test-taker, so as to greatly reduce tension and fatigue during responding, and effectively weaken response tendencies including the social desirability, evaluation anxiety, self-concealment, and central tendency [19]. Third, the imagery gives a deep insight into the individual's subconscious activities. The dual evaluation approach of stable traits and present status can comprehensively and systematically reflect the actual mental state of the individual. This help to accurately assess the situation and find a breakthrough for follow-up treatment. Fourth, the interpretation of the test results reflects the integrity principle of Gestalt theory because it emphasizes the overall evaluation of personality rather than the measurement of single individual traits [19]. At the same time, the purpose of evaluation is to provide effective guidance for treatment rather 
than psychological assessment. Fifth, the imagery is culture-fair and culture-independent to make it possible to use the HIT in cross-culture studies. Last, the HIT can be efficiently used in collective testing as it has relatively fewer items, simple testing procedure and scoring system. The method has been proved to be easy for clinical practitioners. It can be widely used in psychological counseling, employee assistance program, mental evaluation, and other related areas.

\subsection{Limitations of the HIT}

There are some limitations of the HIT. First, when using house imagery technic in counseling settings, the therapist can collect much more details of the client's imagery. Only 12 items referring to the most important features of the house imagery are developed in the HIT in order to make the test easier to be administered. Second, the validity of the HIT for different populations needs more supportive evidence. The psychometric adequacy and clinical contributions of projective methods have been regularly and vigorously challenged, but psychologists regularly use these controversial techniques despite questions regarding their psychometric soundness and utility [20]. In this study, the reliability and validity had been verified using the sample of college students Future studies need to test its reliability and validity in other adult groups, and explore the applicability of the test in the youth population. Third, the HIT only provides limited information of the house imagery. In order to form a detailed individual analysis report and provide feedback, the tester needs more professional training. Above all, the present study shed light into the future projective scales that may combine the theoretic depth and psychometric soundness. Developing a series of imagery projective tests employing other imageries (e.g., flower and insect images) is also the direction worthy of further study.

\section{Conclusion}

The current study suggested it feasible to develop measurements on the basis of theory and practice of Imagery Communication Psychotherapy. Items of this kind of measurements could be designed according to symbolic meanings of imagery. The House Imagery Test had 12 items and showed a stable 4-factor structure, with acceptable reliability and validity. These factors could be labeled as window, appearance, material, and internal article. The House Imagery Test was proved applicable for adults' mental health evaluation. This test might also provide significant information for understanding its underlying causes.

\section{Acknowledgements}

This research was supported by 2016 Academic Advancement Program from School of Sociology and Psychology, Central University of Finance and Economics.

The authors thank those students who spent valuable time with the questionnaires and the teachers who assisted in conducting the investigations.

\section{References}

[1] Dai, X. Y., Zheng, L. X., Ryan, J. J., \& Paolo, A. M. (1993). A survey of psychological tests used in clinical psychological practice of China and its comparison with the data of United States. Chinese Journal of Clinical Psychology, 1 (1), 47-50.

[2] Piotrowski, C. (2015). Projective techniques usage worldwide: A review of applied settings 1995-2015. Journal of the Indian Academy of Applied Psychology, 41 (3), 9-19.

[3] Frank, L. K. (1939). Projective methods for the study of personality. The Journal of Psychology, 8 (2), 389-413.

[4] Hunsley, J., \& Bailey, J. M. (1999). The clinical utility of the Rorschach: Unfulfilled promises and an uncertain future. Psychological Assessment, 11 (3), 266-277.

[5] Tong, H. J. (2002). Surveying and expecting three major test technologies in psychology. Journal of Nanjing Normal University: Social Science, (3), 81-88.

[6] Yuan, Y., Cao, M. Y., \& Liu, Y. (2018). Application of Imagery Communication Psychotherapy in projective test. Psychology and Behavioral Sciences. 7 (3), 38-44.

[7] Yuan, Y., Cao, Y., \& Zhu, J. J. (2013). Clinical techniques of Imagery Communication Psychotherapy. Beijing: Beijing Normal University Publishing Group.

[8] Hu, T. T. (2015). Research on the role of confrontation technique of Imagery Communication Therapy in fear emotion regulation. Unpublished master's dissertation. Beijing Forestry University, Beijing.

[9] Li, J. R., \& Liu, H. J. (2005). Imagery conversing technique in psychological counseling of depression. Chinese Journal of Clinical Rehabilitation, 16 (9), 154-155.

[10] Wang, X. L. (2007). The study on the usage of the method of Imagery Communication in personality tests. Unpublished master's dissertation. Beijing Forestry University, Beijing.

[11] Zhang, H. F., \& He, K. (2007). Validity of imagery conversing technique. Journal of Clinical Rehabilitative Tissue Engineering Research, 30 (11), 6056-6058.

[12] Wang, X. D., Wang, X. L., \& Ma, H. (1999). Rating scales for mental health. Beijing: Chinese Mental Health Journal.

[13] Zung, W. W. K., Richards, C. B., \& Short, M. J. (1965). Self-rating Depression Scale in an outpatient clinic: Further validation of the SDS. Archives of General Psychiatry, 13 (6), 508-515.

[14] Zung, W. W. K. (1971). Self-Rating Anxiety Scale. Psyehosomatics, 12 (6), 371-379.

[15] Huang, L., Yang, T. Z., \& Ji, Z. M. (2003). Application of the Positive and Negative Affect Scale in Chinese. Chinese Mental Health Journal, 17 (1), 54-56.

[16] Waston, D., Clark, L. A., \& Tellegen, A. (1988). Development and validation brief measures of positive and negative affect: The PANAS scales. Journal of Personality and Social Psychology, 54 (6), 1063-1070.

[17] Shweder, R. A., \& D'Andrade, R. G. (1979). Accurate reflection or systematic distortion? A reply to Block, Weiss, and Thorne. Journal of Personality \& Social Psychology, 37 (6), 1075-1084. 
[18] Peng, X. F., Liu, Z. J. \& Zi, F. (2011). The projective test design of achievement motivation statement. Psychological Research, 4 (5), 30-34.

[19] Jain, M., Singh, A., Kar, S. K., Weiner, I. B., \& Kuehnle, K. (2017). Projective Assessment of Children and Adolescents. Reference Module in Neuroscience and Biobehavioral
Psychology.

http://dx.doi.org/10.1016/B978-0-12-809324-5.05058-6

[20] Ryan, A., Timothy, H., Bradley, B. (2019). Use of projective techniques in emotional disturbance evaluations. Journal of the American Academy of Special Education Professionals, 6-14. 